\title{
Instrumentos de avaliação da Qualidade de Vida de pessoas jovens e idosas: um estudo de Revisão Sistemática
}

Camilla Virginia Siqueira Rôla ${ }^{1}$; Susanne Pinheiro Costa e Silva ${ }^{2}$; Patrícia Avello Nicola ${ }^{3}$

\begin{abstract}
Resumo: O presente estudo objetivou apresentar os principais instrumentos validados no Brasil para mensuração da qualidade de vida na população jovem e idosa. Teve como proposta executar uma revisão sistemática através do levantamento e análise de publicações na base de dados da SciElo. Foram encontradas 12 referências, das quais selecionaram-se 6. Os instrumentos relatados foram o SF-36, SF-12, FS-6d, WHOQOL 100, WHOQOL-Bref e PSN. A utilização de instrumento para avaliação da qualidade de vida, na população adscrita das Unidades Básicas de Saúde, fornece um perfil mais global das condições econômicas e psicossociais, bem como às expectativas em relação a própria vida, contribuindo para a humanização e efetividade do serviço de saúde. Esses instrumentos para uso na população brasileira são importantes para gestores e profissionais de saúde, na medida em que estabelecem metas a partir das condições sociais e de saúde encontradas.
\end{abstract}

Descriptores: Instrumentos de Avaliação; Qualidade de Vida; Promoção da Saúde.

\section{Instrumentos de avaliação da Qualidade de Vida de pessoas jovens e idosas: um estudo de revisão sistemática}

\begin{abstract}
Resumo: O presente estudo objetivou apresentar os principais instrumentos validados no Brasil para mensuração da qualidade de vida na população jovem e idosa. Teve como proposta executar uma revisão sistemática através do levantamento e análise de publicações na base de dados da SciElo. Foram encontradas 12 referências, das quais selecionaram-se 6. Os instrumentos relatados foram o SF-36, SF-12, FS-6d, WHOQOL 100, WHOQOLBref e PSN. A utilização de instrumento para avaliação da qualidade de vida, na população adscrita das Unidades Básicas de Saúde, fornece um perfil mais global das condições econômicas e psicossociais, bem como às expectativas em relação a própria vida, contribuindo para a humanização e efetividade do serviço de saúde. Esses instrumentos para uso na população brasileira são importantes para gestores e profissionais de saúde, na medida em que estabelecem metas a partir das condições sociais e de saúde encontradas.
\end{abstract}

Descriptores: Instrumentos de Avaliação; Qualidade de Vida; Promoção da Saúde.

\footnotetext{
${ }^{1}$ Mestre em Ciências pela Universidade Federal do Vale do São Francisco, Petrolina, Pernambuco, Brasil.

E-mail: camillavs.1@hotmail.com

2 Doutora em Psicologia. Professora do Mestrado Profissional em Gerontologia. Universidade Federal da Paraíba. João Pessoa, Paraíba, Brasil. ORCID iD: https://orcid.org/0000-0002-9864-3279. E-mail: susanne.pc@ gmail.com.

${ }^{3}$ Patrícia Avello Nicola : Doutora em Engenharia Florestal. Professora do Programa de Pós-Graduação em Ciências da Saúde e Biológicas da Universidade Federal do Vale do São Francisco, Petrolina, Pernambuco, Brasil.

E-mail: patrícia.nicola@univasf.edu.br
} 


\section{Introdução}

A Qualidade de Vida (QV) é denominada como a medida que faltava na área da saúde e apresenta diferentes interpretações, não tendo um conceito fácil e em consonância entre as ciências. A palavra qualidade, segundo o dicionário Aurélio, é uma condição das pessoas que as distingue das outras e lhes determina a natureza (FERREIRA, 2015). Para a definição de vida, incluem-se dois sentidos:

\footnotetext{
"Os gregos não possuíam um termo único para exprimir o que nós queremos dizer com a palavra vida. [...]zoe, que exprimia o simples fato de viver comum aos seres vivos (animais, homens ou deuses) e bios, que indicava a forma ou maneira de viver própria de um indivíduo ou de um grupo (AGAMBEN, 2007, p. 9).
}

O crescente interesse sobre a temática, fez com que a Organização Mundial da Saúde (OMS) reunisse especialistas sobre saúde e QV de diversas regiões do mundo para formar um grupo de estudos dentro de uma perspectiva transcultural (GORDIA et al., 2011). Diante disso, o conceito mais atual e utilizado é o da OMS, sendo a percepção do indivíduo sobre sua posição na vida, no contexto da cultura e sistema de valores nos quais ele vive e em relação aos seus objetivos, expectativas, padrões e preocupações (WORLD HEALTH ORGANIZATION, 1995).

Após a ampliação do conceito de saúde, que passou a ser vista não apenas como a ausência de doenças, e sim o viver em sociedade acompanhado de bem-estar nas mais diversas acepções, tem sido reforçado o uso da qualidade de vida como um conceito necessário na prática de cuidados para os mais diversos grupos de pessoas.

Desse modo, a QV é verificada em várias acepções e relacionada à saúde, moradia, lazer, hábitos de atividade física e alimentação, resultando em fatores que geram uma percepção positiva de bem-estar (ALMEIDA, GUTIERREZ e MARQUES, 2012), determinados por condições mentais, ambientais e culturais (MINAYO et al., 2000). Assim, deve considerar inúmeras variáveis que a compõem e os vínculos entre elas (ALMEIDA, GUTIERREZ e MARQUES, 2012).

Com o intuito de verificar e medir a saúde dos indivíduos, alguns instrumentos estruturados e simplificados têm sido desenvolvidos e testados. Eles devem ser capazes de 
identificar os estados de bem-estar físico, mental e social dos sujeitos, reconhecendo a relação entre qualidade de vida e a saúde, permitindo inclusive o planejamento de ações de promoção da saúde (CAMPOS; RODRIGUES NETO, 2008).

Os instrumentos relacionados à análise de condições gerais da QV enfatizam questões sobre domínios físicos (dor, fadiga, capacidades e limitações), psicológicos (percepção do estado de saúde, depressão, autoestima, ansiedade e imagem corporal), relações sociais (apoio familiar e social, limitações impostas pela sociedade e as relações interpessoais), nível de independência (mobilidade, atividades cotidianas, capacidade para o trabalho) e noções sobre o bem-estar (corporal, emocional, saúde mental e vitalidade) (GORDIA et al., 2011, p. 50).

No que se refere à gestão em saúde, as estratégias que surgem com o intuito de identificar situações, especialmente aquelas relacionadas à saúde das pessoas, são importantes ferramentas auxiliares no planejamento das políticas públicas, contribuindo para mudanças e melhorias dos serviços. Sendo assim, conhecer os instrumentos disponíveis para mensuração da qualidade de vida pode ajudar no fortalecimento de ações direcionadas para o âmbito individual e coletivo.

Partindo deste pressuposto, o presente estudo visa apresentar os principais instrumentos existentes e validados no Brasil para mensuração da qualidade de vida na população. Espera-se que desperte o interesse de profissionais e gestores em saúde, norteando as suas práticas e auxiliando na adoção de medidas que propiciem a promoção da saúde e busquem solucionar problemas existentes.

\section{Método}

O presente estudo teve como proposta executar uma revisão sistemática através do levantamento e análise de publicações em periódicos na base de dados SciELO, com o intuito de identificar instrumentos de avaliação da qualidade de vida validados no Brasil. Os descritores utilizados foram "Instrumentos de Avaliação", "Qualidade de Vida" e "Promoção à Saúde", que foram integrados por meio do operador lógico "and".

O material foi selecionado utilizando os seguintes critérios: pesquisas que abordassem a mensuração da qualidade de vida, disponíveis na íntegra; artigos científicos, dissertações e 
teses na língua portuguesa. Excluíram-se os artigos que não apresentaram textos completos publicados na íntegra e sem validação no país.

$\mathrm{Na}$ base de dados Scientific Eletronic Library Online (SciElo), ao utilizar-se os filtros dos descritores escolhidos, foram encontradas 12 referências, das quais foram selecionadas 6 por terem correlação direta com o tema proposto. Os materiais selecionados foram publicados no período de 1999 a 2015.

\section{Resultados e Discussão}

Todos os estudos selecionados e analisados para a construção desta revisão estão apresentados no Quadro 1. O resultado desta revisão foi constituído por seis artigos científicos selecionados pelos critérios de inclusão anteriormente estabelecidos.

Devido ao objetivo dessa revisão ser a apresentação de instrumentos validados no País e sua aplicabilidade, discutiremos os materiais encontrados, que trazem as escalas WHOQOL100, WHOQOL-Bref, SF-36, SF12, SF-6D e NHP. Os instrumentos para avaliação da qualidade de vida são comumente modificados de acordo com o objetivo de cada população a ser estudada. Há duas formas de mensurá-la: através de instrumentos genéricos e específicos.

Os instrumentos genéricos abordam o perfil de saúde (população sadia ou com patologia) e estudam indivíduos da população geral ou de grupos específicos (GORDIA et al., 2011), mensurando aspectos funcionais, físicos, dor, estado geral de saúde, vitalidade, aspectos sociais, emocionais e saúde mental (AGUIAR et al., 2008). Já os específicos detectam particularidades da QV em determinadas situações, como as funções físicas, sexual, sono e fadiga (GORDIA et al., 2011). Avaliam as particularidades de uma doença ou agravo, quantificando o impacto do tratamento e os ganhos de saúde obtidos (LANDEIRO et al., 2011).

Geralmente os instrumentos genéricos não avaliam uma patologia específica, mas sim o todo, sem acepção de raça, idade, sexo ou grupo, utilizando questionário para o levantamento e comparação de indivíduos com diferentes patologias. Já os instrumentos específicos aferem de forma concreta grupos exclusivos de pessoas que apresentam em comum algum tipo de patologia, agravo, sexo, faixa etária (VIANNA e CAETANO, 2005). 
Quadro 1 - Descrição dos artigos selecionados para o estudo.

\begin{tabular}{|c|c|c|c|}
\hline Questionário & Título da Publicação & Autor/Ano & Temática \\
\hline $\begin{array}{l}\text { O Medical } \\
\text { Outcomes Study } \\
36 \text { - Item Short - } \\
\text { Form Health } \\
\text { Survey (SF-36) }\end{array}$ & $\begin{array}{l}\text { Tradução para o português } \\
\text { e validação do } \\
\text { questionário genérico de } \\
\text { avaliação de qualidade de } \\
\text { vida "Medical Outcomes } \\
\text { Study 36-Item Short-Form } \\
\text { Health Survey (SF-36). }\end{array}$ & $\begin{array}{l}\text { CICONELLI, R.M } \\
\text { (1997). }\end{array}$ & $\begin{array}{l}8 \text { dimensões da QV: capacidade } \\
\text { funcional, limitação por aspectos } \\
\text { físicos, dor, estado geral de saúde, } \\
\text { vitalidade, aspectos sociais, aspectos } \\
\text { emocionais e saúde mental }\end{array}$ \\
\hline $\begin{array}{l}\text { 12-Item Short- } \\
\text { Form Health } \\
\text { Survey (SF-12) }\end{array}$ & $\begin{array}{l}\text { Avaliação da qualidade de } \\
\text { vida relacionada à saúde } \\
\text { em pacientes com dpoc: } \\
\text { estudo de base } \\
\text { populacional com o SF-12 } \\
\text { na cidade de São Paulo- } \\
\text { SP. }\end{array}$ & $\begin{array}{l}\text { CAMELIER, A.A. } \\
\text { (2005). }\end{array}$ & $\begin{array}{l}12 \text { itens derivados do SF-36, } \\
\text { considerando a percepção do } \\
\text { indivíduo em relação aos aspectos de } \\
\text { sua saúde nas quatro últimas semanas } \\
\text { (SILVEIRA, et al., 2013). }\end{array}$ \\
\hline $\begin{array}{l}\text { Short-Form } 6 \\
\text { dimensions (SF- } \\
\text { 6D) }\end{array}$ & $\begin{array}{l}\text { Validação da versão } \\
\text { brasileira do questionário } \\
\text { genérico de qualidade de } \\
\text { vida short-form } 6 \\
\text { dimensions (SF-6D Brasil) }\end{array}$ & $\begin{array}{l}\text { CAMPOLINA, A.G.; } \\
\text { BORTOLUZZO, A.B.; } \\
\text { FERRAZ; M.B.; } \\
\text { CICONELLI, R.M. } \\
\text { (2011). }\end{array}$ & $\begin{array}{l}\text { Medidas de preferência por estados } \\
\text { de saúde, a partir dos itens do SF-36 }\end{array}$ \\
\hline O WHOQOL-100 & $\begin{array}{l}\text { Desenvolvimento da } \\
\text { versão em português do } \\
\text { instrumento de avaliação } \\
\text { de qualidade de vida da } \\
\text { OMS (WHOQOL-100) }\end{array}$ & $\begin{array}{l}\text { FLECK, M.P.A.; LEAL, } \\
\text { O.F.; LOUZADA, S.; } \\
\text { XAVIER, M.; } \\
\text { CHACHAMOVICH, E.; } \\
\text { VIEIRA, G.; SANTOS, } \\
\text { L.; PINZON, V. (2000). }\end{array}$ & $\begin{array}{l}100 \text { itens referentes a seis domínios: } \\
\text { físico, psicológico, nível de } \\
\text { independência, relações sociais, meio } \\
\text { ambiente e espiritualidade/ } \\
\text { religiosidade/crenças pessoais }\end{array}$ \\
\hline WHOQOL-Bref & $\begin{array}{l}\text { Aplicação da versão em } \\
\text { português do instrumento } \\
\text { abreviado de avaliação da } \\
\text { qualidade de vida } \\
\text { "WHOQOL-bref" }\end{array}$ & $\begin{array}{l}\text { FLECK, M.P.A.; } \\
\text { LOUZADA, S.; } \\
\text { XAVIER, M.; } \\
\text { CHACHAMOVICH, E.; } \\
\text { VIEIRA, G.; SANTOS, } \\
\text { L.; PINZON, V. (2000). }\end{array}$ & $\begin{array}{l}26 \text { itens referentes a quatro domínios: } \\
\text { físico, psicológico, relações sociais e } \\
\text { meio ambiente }\end{array}$ \\
\hline $\begin{array}{l}\text { Perfil de Saúde } \\
\text { de Nottingham ou } \\
\text { Nottingham } \\
\text { Health Profile } \\
\text { (PSN ou NHP) }\end{array}$ & $\begin{array}{l}\text { Adaptação do Perfil de } \\
\text { Saúde de Nottingham: um } \\
\text { instrumento simples de } \\
\text { avaliação da qualidade de } \\
\text { vida }\end{array}$ & $\begin{array}{l}\text { TEIXEIRA-SALMELA, } \\
\text { L.F.; MAGALHÃES, } \\
\text { L.C; SOUZA, A.C; } \\
\text { LIMA, M.C.; LIMA, } \\
\text { R.C.M.; GOULAR, F. } \\
\text { (2004). }\end{array}$ & $\begin{array}{l}38 \text { questões e duas alternativas de } \\
\text { respostas (sim e não), contendo seis } \\
\text { domínios: habilidade física, nível de } \\
\text { energia, dor, reações emocionais, } \\
\text { isolamento social e qualidade do sono }\end{array}$ \\
\hline
\end{tabular}

De acordo com o Quadro 01, o SF-36 é um instrumento do tipo genérico, originalmente na língua inglesa norte-americana, tratando-se de um questionário multidimensional (MATHIAS et al., 2016), avaliado em mais de 200 doenças e traduzido em 40 países (GARRATT et al., 2002). Tem sido empregado em estudos de QV em pacientes com insuficiência renal crônica terminal em hemodiálise (BARBOSA, ANDRADE JÚNIOR e ANDRADE, 2007), hipertensão arterial (SILQUEIRA, 2005), idosos submetidos a cirurgia 
reparadora de fratura do fêmur (MACHADO et al., 2012), portadores de HIV/AIDS (GARCIA et al., 2015), pacientes enteroparasitados (CAVALCANTE, 2015), lombalgia crônica (ADORNO e BRASIL NETO, 2013) e aposentados (PIMENTA et al., 2008).

O SF-12 é internacionalmente reconhecido, curto e compreensível, extensivamente utilizado na avaliação da qualidade de vida relacionada à saúde, monitorando desfechos em saúde tanto em populações gerais quanto específicas (DANTAS; SAWADA; MALERBO, 2003). Utilizado como uma alternativa mais rápida de aplicação do que o instrumento SF-36, sua validade foi evidenciada entre subgrupos populacionais partindo da concepção de qualidade de vida, ou seja, instrumento útil na avaliação do nível de qualidade de vida na população em geral, a partir dos aspectos físicos e mentais (SILVEIRA et al., 2013).

O SF-6D permite a obtenção de medidas de preferência por estados de saúde, a partir dos itens do SF-36, com uma maior praticidade em sua aplicação. Também é considerado uma medida genérica de qualidade de vida, com uso para monitoramento de problemas psicossociais no âmbito individual, percepção de estados de saúde em um grupo da população, auditoria médica e medidas de resultados em serviços de saúde (CAMPOLINA et al., 2011).

O Instrumento de avaliação de QV da OMS foi desenvolvido pelo grupo World Health Organization Quality of Life (WHOQOL), utilizando um enfoque transcultural original, inserindo países de diferentes níveis de industrialização, disponibilidade de serviços de saúde, importância da família e religião dominante. Assim, o objetivo é avaliar a QV geral das pessoas em diferentes culturas (FLECK, et al., 1999).

Ao proporcionar uma avaliação detalhada, o WHOQOL-100 tornar-se muito extenso em estudos epidemiológicos, no qual a avaliação de qualidade de vida é apenas uma das variáveis em estudo. O Grupo de Qualidade de Vida da OMS desenvolveu uma versão abreviada, o WHOQOL-Bref, contendo 26 itens e considerando quatro domínios: físico, psicológico, relações sociais e meio ambiente.

O Perfil de Saúde de Nottingham (PSN), apresenta limitações. A escala não mede todo o contínuo de qualidade de vida, divide os indivíduos em dois níveis de habilidade, apontando ser mais útil quando aplicado em indivíduos mais debilitados. O que este instrumento observa é o efeito-teto para indivíduos de habilidade mais alta e itens fáceis demais para os participantes. Em virtude da baixa complexidade das perguntas e respostas, a utilização desse deve estar associada a outros instrumentos, a exemplo de uma avaliação funcional e/ou uma entrevista (TEIXEIRA-SALMELA et al., 2004). 
Os instrumentos de avaliação da QV discutidos são os mais utilizados no Brasil (ALVES, 2011). Instrumentos como o SF-36 e o WHOQOL da OMS, apresentam vantagens porquê já tiveram sua validade e qualidades psicométricas atestadas, além de permitirem a comparação com outros estudos (ALMEIDA, GUTIERREZ e MARQUES, 2012).

Os instrumentos da Organização Mundial de Saúde (WHOQOL) apresentam considerável melhoria na tradução de seus itens de avaliação em saúde, com uma metodologia diferenciada (revisão por grupo monolíngue e bilíngue), apresentando vantagens em relação àquelas de tradução-retro tradução (OMS, 1997).

Diante do exposto, é necessário que os gestores municipais e estaduais mantenham uma reflexão sobre o conceito de qualidade de vida e de como ela pode ser mensurada, no sentido de estabelecer tais instrumentos em Unidades Básicas de Saúde, otimizando o serviço e conhecendo melhor a área adscrita, bem como os problemas encontrados.

Os questionários validados no Brasil são disponibilizados gratuitamente pela comunidade acadêmica científica. Verifica-se também que não há necessidade de contratação de recursos humanos para realizar esses instrumentos, apenas o apoio dos profissionais de saúde da atenção básica e o incentivo dos gestores.

\section{Conclusão}

Esse estudo identificou os principais instrumentos validados no Brasil e sua importância em mensurá-lo para a melhoria da saúde e da qualidade de vida da população. Os diferentes modelos dos instrumentos apresentam correlações entre si, sugerindo a aplicação destes em diferentes análises em saúde, melhorando e otimizando o serviço de saúde e a própria qualidade de vida, a partir do que seja encontrado, que poderá fornecer subsídios para o planejamento de estratégias de enfrentamento.

A utilização da avaliação da qualidade de vida relacionada à saúde, na população adscrita na UBS, fornece um perfil mais global das condições econômicas e psicossociais, bem como às expectativas em relação a própria vida, contribuindo para a humanização e efetividade do serviço de saúde. 
A partir dos estudos levantados sobre as escalas de qualidade de vida mais utilizadas na população brasileira, são recomendadas todas as escalas apresentadas: SF-36, SF-12, SF-6d, WHOQOL-100, WHOQOL-Bref, Perfil de Saúde de Nottingham.

É importante que os gestores incentivem o uso de tais ferramentas nas unidades de saúde dos municípios, propiciando a melhoria da qualidade de vida a partir de sua mensuração, estabelecendo metas entre os profissionais de saúde. Tais ações poderão otimizar o serviço de saúde, bem como o conhecimento da realidade das condições sociais e de saúde da população.

\section{Referências}

ADORNO, M. L. G. R.; BRASIL NETO,. Avaliação da qualidade de vida com o instrumento SF-36 em lombalgia crônica. Acta Ortopédica Brasileira, São Paulo, v. 21, n. 4, Jul-Ago 2013. ISSN 1413-7852. Disponivel em: <http://dx.doi.org/10.1590/S1413-78522013000400004 >. Acesso em: 10 agoato 2018.

AGAMBEN, G. Homo Sacer: O poder saberano e a vida nua. Tradução de Henrique Burigo. 2. ed. Belo Horizonte: UFMG, 2007. 9-203 p. ISBN 85-7041-307-6.

AGUIAR, C. C. et al. Instrumentos de Avaliação de Qualidade de Vida Relacionada à Saúde no Diabetes Melito. Arquivos Brasileiros de Endocrinologia \& Metabologia, v. 52, n. 6, p. 931-939, 2008. ISSN 1677-9487. Disponivel em: <http://dx.doi.org/10.1590/S0004$27302008000600004>$. Acesso em: 09 agosto 2018.

ALMEIDA, M. A. B.; GUTIERREZ, G. L.; MARQUES, R. Qualidade de vida: definição, conceitos e interfaces com outras áreas de pesquisa. São Paulo: Escola de Artes, Ciências e Humanidades - EACH/USP, 2012. 142 p. ISBN 978-85-64842-01-4.

BARBOSA, L. M. M.; ANDRADE JÚNIOR, M. P.; ANDRADE, K. Preditores de Qualidade de Vida em Pacientes com Doença Renal Crônica em Hemodiálise. Jornal Brasileiro de Nefrologia, v. 29, n. 4, p. 222-229, Dezembro 2007. Disponivel em: <http://www.jbn.org.br/export-pdf/132/29-04-07.pdf>. Acesso em: 10 agosto 2018.

CAMPOLINA, et al. Validação da versão brasileira do questionário genérico de qualidade de vida short-form 6 dimensions (SF-6D Brasil). Ciências \& Saúde Coletiva, Rio de Janeiro, v. 16, n. 7, p. 3103-3110, Julho 2011. ISSN 1413-8123. Disponivel em: <http://dx.doi.org/10.1590/S1413-81232011000800010>. Acesso em: 06 agosto 2018.

CAMPOS, M. O; RODRIGUES NETO, J. F. Qualidade de vida: um instrumento para promoção de saúde. Revista Baiana de Saúde Pública. v.32, n.2, p.232-240 maio/ago. 2008. 
CAVALCANTE, U. M. B. Avaliação da qualidade de vida de pacientes enteroparasitados por meio de um instrimento genérico (SF-36) [Dissertação]. Universidade Federal da Paraíba, João Pessoa, 2015. 69p. Disponivel em: <http://tede.biblioteca.ufpb.br:8080/handle/tede/9067>. Acesso em: 10 agosto 2017.

DANTAS, R. A. ; SAWADA, ; MALERBO,. Pesquisas sobre qualidade de vida: Revisão da produção científica das universidades públicas do estado de São Paulo. Revista Latino Americana de Enfermagem, v. 11, n. 4, p. 532-538, jul-ago 2003. Disponivel em: <https://www.revistas.usp.br/rlae/article/viewFile/1798/1845>. Acesso em: 09 agosto 2018.

FERREIRA, A. B. D. H. Dicionário Aurélio da Lingua Portuguesa. 5a . ed. São Paulo: Positivo Editora, v. 1, 2015.

FLECK, M. P. A. et al. Desenvolvimento da versão em português do instrumento de avaliação de qualidade de vida da OMS (WHOQOL-100). Revista Brasileira de Psiquiatria, São Paulo, v. 21, n. 1, Jan-Mar 1999. ISSN 1516-4446. Disponivel em: <http://dx.doi.org/10.1590/S151644461999000100006 >. Acesso em: 06 agosto 2018.

GARCIA, F. H. et al. Análise da qualidade de vida segundo o questionário SF-36 de pacientes soropositivas e soronegativas em dois ambulatórios do município de Aracaju. Interfaces Científicas - Saúde e Ambiente, Aracaju, v. 4, n. 1, p. 63-69, outubro 2015. Disponivel em: <http://dx.doi.org/10.17564/2316-3798.2015v4n1p63-69>. Acesso em: 10 agosto 2018.

GARRATT, A. et al. Quality of life measurement: bibliographic study of patient assessed health outcome measures. BMJ Journals, v. 324, n. 7351, p. 1417, Junho 2002. Disponivel em: <https://www.ncbi.nlm.nih.gov/pmc/articles/PMC115850/>. Acesso em: 07agosto 2018.

GORDIA, A. P. et al. Qualidade de vida: contexto histórico, definição, avaliação e fatores associados. Revista Brasileira de Qualidade de Vida, Ponta Grossa, v. 3, n. 1, p. 40-52, janjun 2011. Disponivel em: <https://periodicos.utfpr.edu.br/rbqv/article/view/812>. Acesso em: 18 agosto 2018.

LANDEIRO, G. M. B. et al. Revisão sistemática dos estudos sobre qualidade de vida indexados na base de dados SciELO. Ciências \& Saúde Coletiva, Rio de Janeiro, v. 16, n. 10, 2011. ISSN 1413-8123. Disponivel em: <http://dx.doi.org/10.1590/S1413$81232011001100031>$. Acesso em: 08 agosto 2018.

MACHADO, et al. Avaliação da qualidade de vida em idosos pós- fratura da extremidade proximal do fêmur. Arquivos Brasileiros de Ciências da Saúde, v. 37, n. 2, p. 70-75, maio/ago 2012. Disponivel em: <http://files.bvs.br/upload/S/1983-2451/2012/v37n2/a3053.pdf>. Acesso em: 10 agosto 2018.

MATHIAS , et al. Validac ão de questionários para avaliac são da qualidade de vida relacionada à continência fecal em crianc, as com malformac,ões anorretais e doenc,a de Hirschsprung. Revisa Paulista de Pediatria, v. 34, n. 1, p. 99-105, 2016. Disponivel em: <: http://dx.doi.org/10.1016/j.rppede.2015.06.022>. Acesso em: 10 agosto 2018. 
MINAYO, M. C. ; HARTZ , Z. M. A.; BUSS ,. Qualidade de vida e saúde: um debate necessário. Ciência \& Saúde Coletiva, v. 5, n. 1, p. 7-18, 2000. ISSN 1413-8123.

OMS. The World Health Organization quality of life assesment (WHOQOL). Position paper from the word The World Health Organization, 41, n. 10, 1997. 1403-1049.

PIMENTA, A. et al. Avaliação da qualidade de vida de aposentados com a utilização do questionário SF-36. Revista da Associação Médica Brasileira, v. 54, n. 1, p. 55-60, 2008. ISSN 0104-4230. Disponivel em: <http://dx.doi.org/10.1590/S0104-42302008000100021〉. Acesso em: 10 agosto 2018.

SILQUEIRA, S. M. D. F. O questionário genérico SF-36 como instrumento de mensuração da qualidade de vida relacionada a saúde de pacientes hipertensos [TESE]. Escola de

Enfermagem de Ribeirão Preto, Ribeirão Preto, 2005. Disponivel em:

<10.11606/T.22.2005.tde-17052007-160822>. Acesso em: 10 agosto 2018.

SILVEIRA, et al. Propriedades psicométricas do instrumento de avaliação da qualidade de vida: 12-item health survey (SF-12). Ciências \& Saúde Coletiva, Rio de Janeiro , v. 18, n. 7, p. 1923-1931, Julho 2013. Disponivel em: <http://dx.doi.org/10.1590/S1413-

81232013000700007>. Acesso em: 10 agosto 2018.

TEIXEIRA-SALMELA, et al. Adaptação do Perfil de Saúde de Nottingham: um instrumento simples de avaliação da qualidade de vida. Cadernos de Saúde Pública [online], v. 20, n. 4, p. 905-914, 2004. ISSN 1678-4464. Disponivel em: <http://dx.doi.org/10.1590/S0102311X2004000400004>. Acesso em: 08 agosto 2018.

VIANNA, C. M. ; CAETANO,. Avaliações econômicas como um instrumento no processo de incorporação tecnológica em saúde. Cadernos de Saúde Coletiva, Rio de Janeiro, v. 13, n. 3, p. 747-766, 2005. Disponivel em: <http://www.cadernos.iesc.ufrj.br/cadernos/images/csc/2005_3/artigos/CSC_2005-

3_cid.pdf $>$. Acesso em: 09 agosto 2018.

WORLD HEALTH ORGANIZATION. The World Health Organization quality of life assesment (WHOQOL). Position paper from the word The World Health Organization, 41, n. 10, 1995. 1403-1049..

Como citar este artigo (Formato ABNT):

SIQUEIRA RÔLA, Camilla Virginia; SILVA, Susanne Pinheiro Costa e; NICOLA, Patrícia Avello. Instrumentos de avaliação da Qualidade de Vida de pessoas jovens e idosas: um estudo de revisão sistemática. Id on Line Rev.Mult. Psic., 2018, vol.12, n.42, p. 111-120. ISSN: 1981-1179.

Recebido: 22/08/2018;

Aceito: 25/08/2018 